\title{
Skin Test for Cutaneous Adverse Drug Reactions
}

\author{
Yusuf Wibisono, Damayanti \\ Departement of Dermatology and Venereology, Faculty of Medicine Universitas Airlangga/Dr. \\ Soetomo General Academic Teaching Hospital Surabaya
}

\begin{abstract}
Background: The incidence of drug eruptions is increasing during the last few years due to a large number of new medications. Early detection of the causative agent and the prevention from exposure are crucial managements in terms of drug eruption, mainly to prevent its recurrence. Objective: To understand skin test as a diagnostic modality in drug eruption, which includes skin patch test, skin prick test, and intradermal test. Literature review: Drug eruption is a form of skin eruption triggered by the use of medications, topical or systemic, in the right dose and indication. The manifestation can vary from maculopapular, urticaria, pustular, and bullous eruption; from the most nonsignificant to a life-threatening reaction. A diagnostic procedure is critical to discover the type of drugs that cause the eruption, i.e., skin test, specific IgE measurement, histamine-release test, and provocation test. Skin test is the first choice in the diagnostic process as it is simple, easy, practical and has high sensitivity and specificity. Conclusion: Skin test is one of the many available diagnostic tools. However, both false positive and false negative results might still arise. The experts are currently attempting to come up with more accurate and practical tests to aid the diagnostic of drug eruption, thus preventing its occurrence.
\end{abstract}

Keywords: Drug eruption, skin test.

Correspondence address: Damayanti, Department of Dermatology and Venereology, Faculty of Medicine, Universitas Airlangga, Dr. Soetomo General Academic Teaching Hospital, Jl. Prof. Dr. Moestopo No. 6-8 Surabaya 60131, Indonesia. Phone: +62315501609. Email: dr_damayanti_bs@yahoo.com.

\section{INTRODUCTION}

The incidence of drug eruption is increasing during the past few years due to a large number of new drugs. Its clinical manifestations are mostly trivial, although about $20 \%$ of the cases are severe and require distinctive treatment in a hospital. In the Dermatology Department, drug eruption accounts for about 1-2\% case in the outpatient division and $5-10 \%$ case in the inpatient division. ${ }^{1-3}$

Early detection of the cause and prevention from its exposure are critical managements in drug eruption, mainly to prevent the recurrence of potentially worsening the eruption. ${ }^{1,4,5}$ Additional diagnostic tests are skin test, specific IgE measurement, histaminerelease test, and drug provocation test, which is the gold standard in diagnosing the triggering drugs. However, drug provocation test as the current gold standard is still under dispute it may introduce lifethreatening risk..$^{6,7,8}$

The skin test is a simple, easy, snd practical test, and it offers high sensitivity and specificity. The procedure also carries less risk and rarely fatal compared to drug provocation. This article covers skin patch test, skin prick test, and intradermal test. . $^{1,3,9,10}$

\section{LITERATURE REVIEW}

According to $\mathrm{WHO}$, adverse drug reaction is a noxious and unintended response or reaction to a drug that occurs at doses normally used for prophylaxis, diagnosis, or therapy of disease or the modification of physiologic function. Cutaneous adverse drug reactions (CADR) or drug eruption is a form of skin eruption triggered by drug usage, topical or systemic, at the right dose and indication. ${ }^{2,3}$

Based on its pathomechanism, adverse drug reaction is grouped into five types; type A (augmented), which is an exaggerated pharmacological activity of the medication; type B (bizarre), an unpredicted effect, idiosyncrasy, and usually related to the immune system; type $\mathrm{C}$ (chemical), which is the chemical/pharmacological effect of the substance; type D (delayed), a delayed effect of the substance, usually related to a carcinogenesis or teratogenesis effect; and type E (end-of-dose), a reaction to the drug's suspension. $^{2}$

The most common adverse reaction is the dosedependent type A, which can be predicted through the primary and secondary pharmacology of the drug. Meanwhile, type B is not quite predictable; as it is affected by tha specific vulnerability of particular subject or genetic. ${ }^{3}$ 
The type B adverse drug reaction can manifest in the form of IgE-mediated type I hypersensitivity, which occurs right after exposure, or in the form of $\mathrm{T}$ cells-mediated type IV hypersensitivity (delayed type). Therefore, based on the time of the onset, drug eruption is divided into early/immediate reaction that occurs within 1-2 hours after exposure, and delayed reaction that occurs after several hours, days, up to 6 weeks after drug exposure. ${ }^{2}$

The manifestation of drug eruption varies greatly, from a nonspecific occurrence such as maculopapular lesion or angioedema that is frequently found in the immediate type reaction to pustular lesion, bullous eruption, maculopapular exanthema, exfoliative erythrodermal, vasculitis, lichenoid reaction or subacute lupus erythematous, Stevens Johnson Syndrome (SJS), Toxic Epidermal Necrolysis (TEN), Fixed Drug Eruption (FDE), and acute generalized exanthematous pustulosis (AGEP) that usually occur in the delayed type. ${ }^{2,3}$

Drug eruption is oftentimes a different entity of disease; therefore, it needs a systematic approach, just as other skin diseases do. An accurate analysis of the pattern of the eruption greatly aids in discovering the causative substance, considering that different types of drugs are usually related to various types of reaction..$^{2,3}$

Analyzing the causative drug is crucial, i.e., skin test, specific IgE measurement, histamine release test, and provocation test. Skin test is the first choice of the diagnostic tool due to its simple, easy, and practical procedure, as well as its high sensitivity and specificity. ${ }^{1,9,10}$

Rive, Bourke, and Philips (2013) stated that skin testing in penicillin-related drug eruption has $70 \%$ sensitivity and $97-100 \%$ specificity, while in cephalosporin-related drug eruption, the sensitivity varies from $30,7 \%$ to $69,7 \%$ due to the wide choices of cephalosporin. Skin testing in neuromuscular blocking agents (NMBA) has $94-97 \%$ sensitivity. ${ }^{6}$

Skin testing is safer and less known to cause fatal side effects compared to oral provocation test. In terms of drug eruption, commonly used skin tests are skin patch test, skin prick test, and intradermal skin test. $^{1,3,9,10}$

The skin patch test for drug eruption is thriving these past few years due to its easy and relatively safe procedure. However, this test is not commonly done as a systematic investigation in larger centers due to its low sensitivity (11-38\%)., ${ }^{2,3}$

The basic principle of the skin patch test is T cellmediated type IV hypersensitivity. The adaptive immune system activates $\mathrm{T}$ cells due to the formation of hapten-Antigen Presenting Cell (APC) and Human Leukocyte Antigen (HLA) complex. The drug molecules or its metabolites act as a hapten that bounds to proteins, thus forming an antigen that will be processed by the APC. APC-processed antigens activate $\mathrm{T}$ lymphocytes, triggering their proliferating cycle and producing a colony of specific memory $\mathrm{T}$ lymphocyte or effector $\mathrm{T}$ lymphocyte. Activated $\mathrm{T}$ cells activate $\mathrm{B}$ lymphocyte to produce antibodies (IgA, IgG, and $\operatorname{IgE}$ ). It takes about 7-10 days until this immunological memory is fully formed (sensitization phase). This phase requires continuous and sufficient exposure to the antigen for the tissue or organ damaging hypersensitivity reaction to occur (elicitation phase). Re-exposure to certain (presumably) causative drugs will allow an identical clinical reaction pattern in sensitized patients. This is the base of skin patch test in drug eruption. ${ }^{3,11}$

An ideal skin patch test bears neither false positive nor false-negative results. The test itself is expected to have as little detrimental effect as possible, particularly in terms of side effects and without sensitization to patch tests. The skin patch test is expected to have good positive predictive value (PPV), which is the real number of diseased individuals among positive test results (in percentage) in a population. It is also expected to have good negative predictive value (NPV), which is the true number of non-diseased people among negative test results in a population. . $^{2,3,11}$

Both the positive and negative predictive values depend on a number of parameters that are unrelated to sensitivity, which is defined as the probability of positive results in a diseased subject. Specificity is defined as the probability of negative results in a healthy subject. ${ }^{2,3,11}$

An ideal screening test is reliable, accurate, and has a good intraobserver as well as reproducible interobserver value. While the patch test utilizes optimal concentration and vehiculum, it is-just as many other diagnostic tools - not $100 \%$ sensitive nor specific. Although limited to some degree, the reliability of a patch test, can be increased and supported by additional tests such as semi-open test, Repeated Open Application Test (ROAT), and a number of other methods to assess the clinical relevance of the patch test. ${ }^{2,3,11}$

An allergen's bioavailability parameters can interact with each other, namely the intrinsic penetrating capacity, concentration, vehiculum, the occlusivity of the patch test, and the plaster, as well as the exposure time. Several allergens are insoluble in petrolatum due to its chemical instability; therefore, the allergen is available in a water-based solution., ${ }^{2,3}$

An ideal patch test includes a thoroughly preparat-covered area that does not overflow outside the testing field; to ensure accurate, non-overlapping 
interpretation. Gomcalo and Bruynzeel (2010) in their study mentioned that the optimal dose of pet preparat in Finn Chamber is $20 \mathrm{mg}$, while the minimum dose of $35 \mathrm{mg}$ is suggested in van der Bend Chamber. ${ }^{2,3}$

The same requirement applies to the liquid volume. Mahajan and Handa (2013) strongly suggested the use of the Finn Chamber for liquid samples. The recommended volume is 15 or $20 \mu \mathrm{L}$; and should be measured with a calibrated pipette. The van der Bend Chamber is the right choice for acetone and ethanol; with $20 \mu \mathrm{L}$ liquid. The chamber is applied right onto the skin to prevent evaporation. This study used the van der Bend Chamber, and there were no signs of irritation from acetone and ethanol. ${ }^{2,3}$

The patch test uses standardized allergens appropriate to the presumably causative agents. It is suggested to conduct the Repeated Open Application Test (ROAT) with control should there be doubts in determining the causing agents. The test should not use allergens with an unknown concentration, and the patients should be $>20 \mathrm{mg} /$ day of steroid-free for at least two weeks prior. The testing area should be steroid- and topical immunosuppressant-free for at least three days prior. The targeted skin should be clear from any lesion. A lesion of $>25 \%$ body surface area might cause Angry Back Syndrome. The subjects should not consume any immunosuppressant agents, such as methotrexate, cyclosporine, azathioprine, or mycophenolate mofetil prior to testing; as they might induce type IV hypersensitivity reaction. Another requirement for patch test is no history of allergy to plaster and no signs of sunburn. The systemic effect of UVB/UVA might cause false-negative result despite the indirect irradiation. Antihistamines are not known to affect standardized patch test results. ${ }^{2,3}$

The test is best done on the upper back or the outer part of the upper arm region. Other regions often bear false-negative results. To avoid a false-positive result, each chamber must be spaced for at least $15 \mathrm{~mm}$. The procedure should also consider medicolegal aspects as well as the allergen's concentration for children and pregnant women. ${ }^{2,3}$

The patch test takes 5-7 days to complete. If only one to two allergens are used, the test is done on the outer part of the upper arm, while the use of multiple allergens with Finn chamber should be done with Finn chamber kit on the upper back. The targeted skin area should be free from nevi or seborrheic keratoses. For hairy skin, hair plucking should be done beforehand; and given one to two days for the following irritation to subside in case irritation occurs. The procedure is done either in a sitting or prone position. First, the patch test unit is filled with allergens and labeled. The targeted skin area is cleansed with alcohol $70 \%$ and left to dry. The patch test unit is then applied with a hypoallergenic plaster and left for 48 hours. ${ }^{2,3}$

The patient is allowed to go home, noting that the test area should always be dry. They should not wet the test area, refrain from sweat-triggering activities, withhold from scratching red or itchy area, prevent friction, and avoid sunrays/UV. After 48 hours, they should come back to have the patch removed. The removal process should not involve alcohol. The physician should wait for 30 minutes before starting the interpretation. If the subject feels overwhelmed by unbearable itchiness or pain, they are allowed to remove the patch themselves (mainly in nonstandardized allergen) without disrupting occluded adjacent chambers. ${ }^{2,3}$

The reading is done on the $48^{\text {th }}, 72^{\text {nd }}$, and $96^{\text {th }}$ hour or up to 5 days after removal. The area is either marked with a certain marker or mapped on the testing site to facilitate the next day interpretation. The test result is documented and photographed in each reading. Any complaint of pain during the test should be reported to the physician. ${ }^{2,3}$
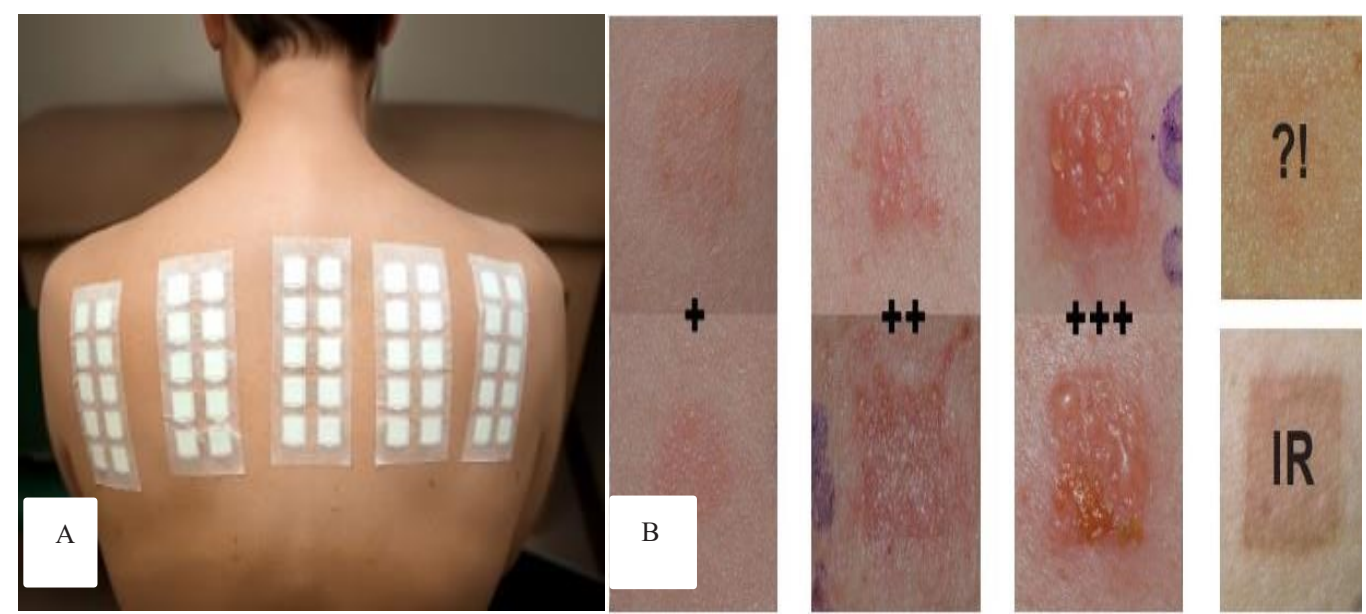

Figure 1. Picture A. The patch test procedure according to CDRG. Picture B. The results of a patch test according to $\mathrm{CDRG}$. 
It is suggested that the patch test utilizes the pure form of a substance, and if possible, in either petrolatum, water, or alcohol between 1-10\% concentration. Liquid or powder preparat acquired from ground capsules or tablets and pills can be used with up to $30 \%$ concentration. The use of water or alcohol is more recommended instead of petrolatum to avoid a false-negative reaction. A control sample is important in predicting false positive result as well as eliminating irritation-triggered reaction. ${ }^{1,2,3}$

The European Society of Contact Dermatitis suggests six weeks to 6 months time span after the total resolution of the preceding drug eruption. The consumption of corticosteroids and other immunosuppressant agents should be stopped for at least two weeks before the test. ${ }^{1,3,12}$

Topical corticosteroids should not be applied to the targeted skin area for at least two weeks prior. A larger dose of topical corticosteroids should also be avoided, although it is not applied directly to the testing area due to its similar effect with low-dose systemic corticosteroids. Intense UV exposure, such as prior sunbathing, might also lower the test's reactivity. ${ }^{1,3}$

Not all types of skin lesion in drug eruption gives positive result in a patch test. The test will bear positive results if the pathomechanism of the drug eruption includes delayed type hypersensitivity reaction (type IV). The patch test is mainly indicated for drug eruptions in the form of PEGA ( $\geq 50 \%$ sensitivity), anticonvulsant hypersensitivity syndrome $(70 \%$ sensitivity), DRESS ( $\geq 72 \%$ sensitivity), SJS-TEN (near 100\% sensitivity), beta-lactam group antibiotics (39-54\% sensitivity), NSAID-related FDE (40-87\% sensitivity), contact/systemic contact dermatitis and photocontact ( $\geq 87 \%$ sensitivity). However, the patch test is less meaningful in a number of cases, i.e., immediate drug reaction, pruritus, vasculitis, several cases of SJS-TEN (e.g., due to Allopurinol), and systemic drug-related photodermatitis. ${ }^{2,3}$

The result-reading is done on the second, fourth, and seventh day. It is scored according to the International Contact Dermatitis Research Group (ICDRG) criteria, as shown in Table 1. A false-positive result might be due to the irritating effect of direct substance applied to the skin, while false negative result might be due to one of two major causes, i.e., insufficient penetration to trigger an allergic response; or the metabolite of a systemic drug-instead of the drug molecule - acts as the causative allergen, which will reach the skin after going through the metabolism process. Another explanation to a false negative result is the incorrect time-choosing, the drug reaction is not due to the immune system, the absence of causative factor such as viral infection, and other drugs that trigger the immune system. ${ }^{1,2,3}$

Table 1. Scoring system for patch test according to ICDRG ${ }^{1}$

\begin{tabular}{cc}
\hline Score & Interpretation \\
\hline- & Negative reaction \\
++ & Doubtful reaction; faint erythema only \\
+ & Strong (edematous or vesicular) test reactions; erythema, infiltration, vesicles \\
++ & Extreme (bullous or ulcerative) \\
+++ & Irritant reactions of different types \\
IR & Not tested \\
NT &
\end{tabular}

A cross-reaction might occur in a patch test, e.g., Amoxicillin that always causes a cross-reaction with Ampicillin. However, this cross-reaction is rarely found with Benzylpenicillin or Carbopenem and Cephalosporin. Such a reaction is also frequently found between Cephalosporins and between Fluoroquinolones. However, a cross-reaction in a patch test is not always associated with the result of the oral provocation test. Patch tests rarely cause side effect, although it usually corresponds with an anaphylactic reaction, namely Penicillins, Neomycins, and Bacitracin. $^{2}$

The second, skin prick test, was initially introduced by Blackley in 1865. Its use as a diagnostic tool was first published in a study by Helmtraud
Ebruster in 1959. The skin prick test is a reliable method to diagnose IgE-mediated allergy in rhinoconjunctivitis, asthma, urticaria, anaphylactic, atopic eczema patients, as well as suspected food or drug allergy. ${ }^{13,14}$

Skin prick test is easier to do, less invasive, relatively cheaper, require less time, and reproducible with the same result if skilled health workers do it. The skin prick test offers good specificity and sensitivity (70-95\% and $80-97 \%$, respectively) to detect allergic reactions due to inhaled allergen. However, in terms of drug eruption, both its sensitivity and specificity vary; depending on the type of drug. For example, the NPV in Penicillin reaches $98,5 \%{ }^{13,14}$ 
Skin prick test is used to confirm a type I allergy reaction. It is used to diagnose drug eruptions, mainly in urticarial drug reactions or other drug-induced lesions as the result of a combination of type I hypersensitivity with other types reaction. ${ }^{1}$

The skin prick test is done by applying a small amount of allergen on to the dermis. In allergic subjects' skin, the immune system's effector cells that hold specific IgE for certain allergens will be activated after exposure to the allergen, thus triggering mediators (histamine, tryptase, TNF- $\alpha$, prostaglandin, leukotriene, IL-4, etc.) release. This release induces vasodilation and an increase in skin vascular permeability, thus causing tissue edema, "wheal reaction", and local erythema. In skin prick test, the wheal or the local swelling size will determine whether the test turns out to be positive or negative, while erythema is usually not taken into account. A delayed phase reaction may occur in the next one to two hours, peaks within 6 to 12 hours after exposure, and recedes within 48 hours. ${ }^{14}$

If possible, an ideal skin prick test should be done at least 4-6 weeks after a systemic allergic reaction. Antihistamines falter its sensitivity; thus, it is strongly suggested to stop antihistamines consumption three days prior. Ketotifen should be stopped 15 days prior to the test. Oral methylprednisolone for more than $8 \mathrm{mg}$ a day and other equivalent dose of corticosteroids will also affect the result. NSAIDs and topical corticosteroids may also affect the result, although it is still in dispute. ${ }^{11,15}$

Skin prick test should not be done when a relapse is taking place (the subject should be lesion-free for at least two weeks). The subject should be antihistaminesfree for three days, the targeted test area should be topical corticosteroids-free for seven days, and any systemic corticosteroid, immunosuppressant, and immunomodulatory agents consumption should be stopped during the test. This test should not be done to pregnant women. ${ }^{11,13,15}$

The skin prick test is done on the upper back (for infants, due to its higher sensitivity) or on the volar plate of the lower arm. The skin should be disinfected with alcohol $70 \%$ and let dry (do not wipe). Using a marker and a ruler, mark the skin to form spaces for at least $1,5-2 \mathrm{~cm}$ (ideally $3,5 \mathrm{~cm}$ ) to avoid mixing and overlapping interpretation. A drop of histamine solution (50-100 $\mu \mathrm{M}$ in a saline solution) is then applied as the positive control, another drop of normal saline solution as the negative control, and one drop of each (presumably) causative allergen extract. Next, the physician will prick the skin through the liquid with a $26,5 \mathrm{G}$ or $27 \mathrm{G}$ needle or a blood lancet with prickpuncture test method or modified prick test method. The pricking should not cause bleeding, although a drop of blood is still tolerated. The interpretation can be started after 15-20 minutes.
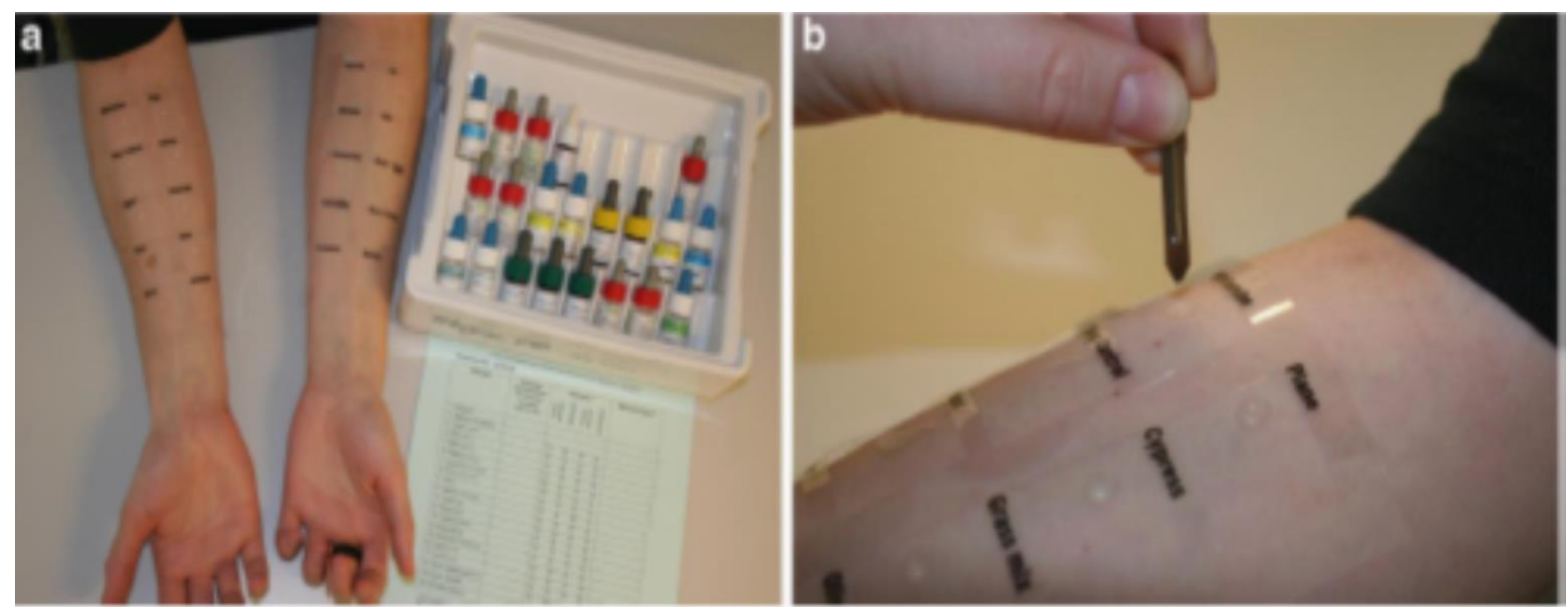

Figure 2. Picture A. The volar plate of the lower arm is usually used fos testing in adults. Picture B. Once disinfected, a $26 / 5 \mathrm{G}$ or $27 \mathrm{G}$ needle or blood lancet is used to prick the skin.

Both positive and negative control should be read first. The negative control is used to eliminate dermographism that could intervene in the reading. It is a positive result if the wheal diameter is half or more than that of the positive control. ${ }^{13,15,16}$

The test comes out as false negative if the positive control sample bears weak or negative results, inadequate time of interpretation, or consumption of oral antihistamine or corticosteroid beforehand. A false-positive result may occur if the negative control sample bears the positive result, the subject suffers from dermographism, or there is an all-positive result from all reagents in the skin prick test. ${ }^{15,17}$ 


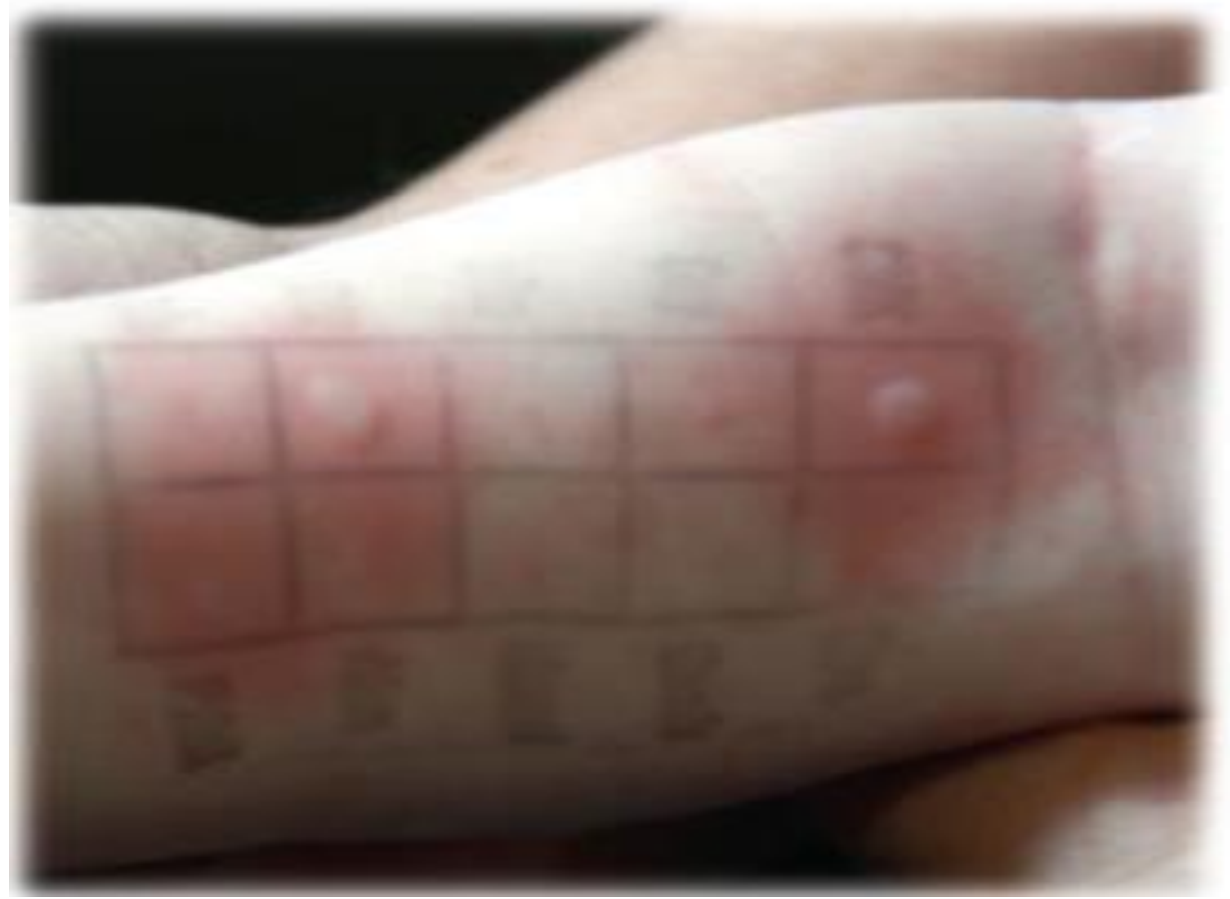

Figure 3. A positive skin prick test result (wheals visible) ${ }^{17}$

The skin prick test is relatively safe; there has not been a report of a fatal side effect for the last five years in the United States, according to Lachapelle and Maibach (2014). Side effect in the form of acute asthma exacerbation might occur if the test uses respiratory allergen to an asthmatic subject. A fatal side effect might occur in the use of natural substance extract as the allergen. ${ }^{13,14,17}$

Skin prick test is indicated for type I allergy reaction to identify individual sensitivity to certain inhalants, foods, drugs, or occupational allergens (e.g., in latex allergy). It is also indicated for subjects with rhinitis, rhinoconjunctivitis, rhinosinusitis, asthma, allergic conjunctivitis, and atopic dermatitis. It is also appropriate for food-allergic subjects prone to anaphylactic reactions, immediate acute urticaria, and acute eczema. There are no age; limitations; therefore, the test can be done for both children and adults. ${ }^{13}$

Certain types of drugs might affect the resultlessening the accuracy. These types of drug are: antihistamines, glucocorticosteroids, and other systemic medications such as omalizumab, leukotriene receptor antagonists, cyclosporin $\mathrm{A}$, theophylline, antidepressants, and adrenergic agonists. ${ }^{13}$

The skin prick test should not be done in subjects with the following criteria: scattered skin lesion such as severe eczema, severe dermographism, urticaria, uncooperative subjects, as well as those who are unable to stop antihistamines or other intervening drugs' consumption. ${ }^{13,16}$

The third test is the intradermal test, which should only be done if the skin prick test comes out negative after 20 minutes using a (presumably) causative allergen. This test should only be done in a hospital under strict supervision. The intradermal test has a higher sensitivity than the skin prick test. About $26,67 \%$ of cases with negative skin prick test, the intradermal test bears positive result. ${ }^{14,18}$

Kavadas et al. performed a study involving 59 children with a food allergy, in which only one subject had a positive skin prick test, while the other 58 subjects had negative results. The 57 subjects with a skin prick results underwent intradermal test. From those 57 subjects with negative skin prick test results, 11 subjects turned out to have positive intradermal results. This shows that the intradermal test has higher sensitivity compared to that of skin prick test. Both tests share the same basis - the IgE-dependent early hypersensitivity reaction (type I). ${ }^{8,16}$

The intradermal test is indicated for drug eruption cases with exanthematous maculopapular eruption and FDE. It is also indicated for hypersensitivity to insect venom, drug allergy mainly of beta-lactams, cephalosporins, insulin, opium, anesthetics, muscle relaxants, and enzymes. Furthermore, it is indicated to detect immediate hypersensitivity reactions to a number of vaccines. This test is not indicated to test the sensitivity to aeroallergens and foods. ${ }^{16,18}$

The use of certain drugs should be stopped before the test because it might intervene with the reaction or the result, such as antihistamines (H1 blockers and Ketotifen), oral and topical corticosteroids, and topical calcineurin inhibitors. ${ }^{14}$ 


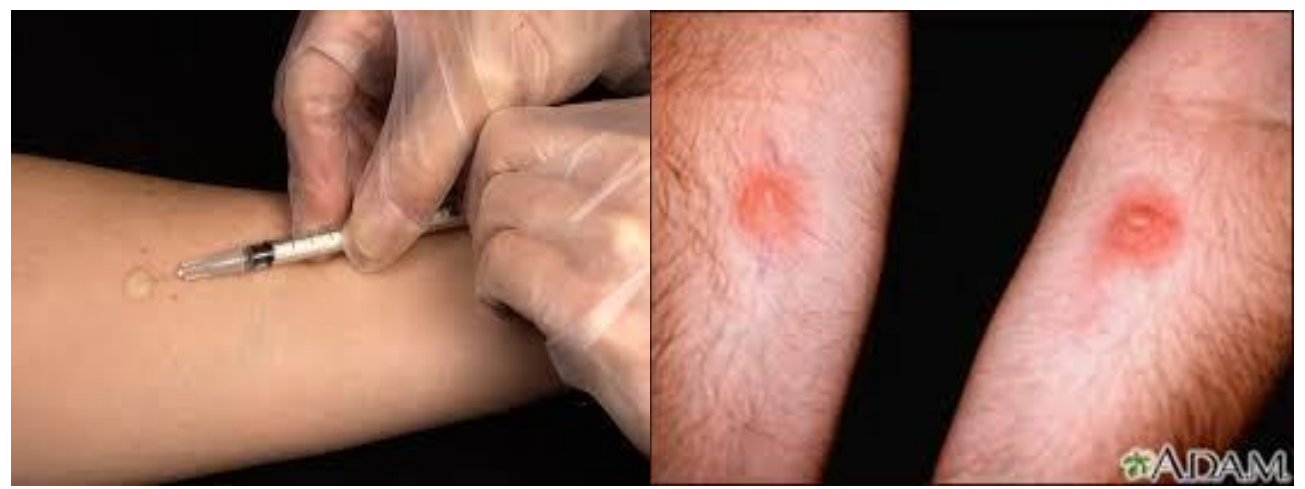

Figure 4. The procedure and positive result of intradermal skin test ${ }^{19,20}$

Experience and expertise of the physician plays a major role in acquiring decipherable results in an intradermal test. Once disinfected with alcohol, the skin is injected with $0,02 \mathrm{ml}$ to $0,05 \mathrm{ml}$ of allergen intradermally, forming a bleb or a small intradermal bubble with approximately $3 \mathrm{~mm}$ in diameter. The injected allergen should be diluted 100 to 1000 times of that used in skin prick test. ${ }^{14,16}$

The interpretation begins after 20 minutes. It is a positive result if the formed wheal is $\geq 6 \mathrm{~mm}$ in diameter. A diameter of $5 \mathrm{~mm}$ and less is a-negative. Barbaud emphasizes the importance of both intradermal test interpretations in 48 and 72 hours after the test, particularly in exanthematous maculopapular eruption due to medications such as beta-lactam antibiotics, synergistin, platinum salt, iodinated contrast media, and heparin. ${ }^{14,19,20}$

The advantage of this test lies in its higher sensitivity compared to the skin prick test, although the intradermal procedure is more painful, more complicated, and often bears false-positive results. Moreover, the possibility of systemic allergic reaction is higher than that of skin prick test, although rarely fatal. ${ }^{14,19,20}$

\section{DISCUSSION}

Diagnosing the causative agent is critical in terms of drug eruption. An example of the diagnostic tools is the skin test. It is relatively easy to perform, relatively cheap, and offers good sensitivity and specificity. There are three skin test options in terms of drug eruption, i.e., skin patch test, skin prick test, and intradermal test. The use of the appropriate tool to the type of occurring reaction will boost the sensitivity and specificity of the skin test.

\section{REFERENCES}

1. Lachapelle JM. Testing procedures in cutaneous systemic immune-related adverse drug reactions. In:Lachapelle JM, Maibach $\mathrm{H}$, editors. Patch testing and prick testing: a practical guide. New York: Springer-Verlag; 2012. p. 173-85.

2. Goncalo M, Bruynzeel DP. Patch testing in adverse drug reactions. In:Johansen JD, editor. Contact dermatitis. Berlin-Heidelberg: SpringerVerlag; 2010. p.1-18.

3. Mahajan VK, Handa S. Patch testing in cutaneous adverse drug reactions: methodology, interpretation and clinical relevance. IJVDL 2013; 79(6):836-41.

4. Thong BYH, Mirakian R, Castells M, Pichler W, Romano A, Bonadonna $\mathrm{P}$, et al. A world allergy organization international survey on diagnostic procedures and therapies in drug allergy/hypersensitivity. WAOJ 2011; 4:257-70.

5. Lehloenya RJ. Cutaneous adverse drug reactions. CME 2011; 29(6):238-42.

6. Rive CM, Bourke J, Phillips EJ. Testing for drug hypersensitivity syndromes. Clin Biochem Rev 2013; 34:15-38.

7. Schnyder B, Pichler WJ. Nonimmediate drug allergy: diagnostic benefit of skin testing and practical approach. J Allergy Clin Immunol 2012; 129:1170-1.

8. Kavadas FD, Kasprzak A, Atkinson AR. Antibiotic skin testing accompanied by provocative challenges in children is a useful clinical tool. AACI Journal 2013; 9(22).

9. McKay D. Cutaneous allergy. J R Coll Physicians Edinb 2013; 43:241-5.

10. Brockow K, Garvey LH, Aberer W, AtanaskovicMarkovic M, Barbaud A, Bilo MB, et al. Skin test concentrations for systemically administered drugs - an ENDA/EAACI drug allergy interest group. EAACI 2013; 68:702-12.

11. Abbas AK. Hypersensitivity. In:Abbas AK, Lichtman AH, Pillai S, editors. Basic immunology. Philadelphia: Elsevier Saunders; 2014. p. 207-25. 
12. Clayton T. Patch testing. [Online].; 2016 [cited 2016 May 23. Available from: http://www.dermexpert.co.uk/patch-testing/.

13. Heinzerling L, Mari A, Bergmann KC, Bresciani M, Burbach G, Darsow U, et al. The skin prick test - European standards. CTA Journal 2013; 3(3).

14. Niederberger V, Eckl-Dorna J, Pauli G. Recombinant allergen-based provocation testing. Methods 2014; 66: 96-105.

15. Lachapelle JM, Maibach HI. Methodology of open (non-prick) testing, prick testing and its variants. In:Lachapelle JM, Maibach HI, editors. Patch testing and prick testing. New York: Springer-Verlag; 2012. p. 159-70.
16. ASCIA. Skin prick testing for the diagnosis of allergic disease : ASCIA 2016.

17. QAS. Causes and testing. [Online]; 2012 [cited 2016 May 23. Available from: http://www.qldallergy.com/causes-and-testing.

18. Mahboob A, Haroon TS, Iqbal Z, Saleemi MA, Munir A. Fixed drug eruption and intradermal provocation test. JCPSP 2008;18(12): 736-9.

19. KCPP. Intradermal testing. [Online].; 2016 [cited 2016 May 23. Available from: http://www.kcallergycenter.com/intradermaltesting.

20. KMoU. Allergy testing - skin. [Online].; 2014 [cited 2016 May 23. Available from: http://keckmedicine.adam.com/content.aspx?pro ductId $=117 \&$ pid $=1 \&$ gid $=00519$ 